\title{
New Active Galactic Nuclei Among the INTEGRAL and SWIFT X-ray Sources
}

\author{
R. A. Burenin ${ }^{* *}$, A. V. Mescheryakov ${ }^{1}$, M. G. Revnivtsev ${ }^{1,2}$, \\ S. Yu. Sazonov ${ }^{1,2}$, I. F. Bikmaev ${ }^{3,4}$, M. N. Pavlinsky ${ }^{1}$, and R. A. Sunyaev ${ }^{1,2}$ \\ ${ }^{1}$ Space Research Institute, Russian Academy of Sciences, Profsoyuznaya ul. 84/32, Moscow, 117997 Russia \\ ${ }^{2}$ Max-Planck-Institut für Astrophysik, Garching, Germany \\ ${ }^{3}$ Kazan State University, Kremlevskaya ul. 18, Kazan, 420008 Russia \\ ${ }^{4}$ Academy of Sciences of Tatarstan, Kazan, 420111 Russia \\ Received December 26, 2007
}

\begin{abstract}
We present the results of our optical identifications of a set of X-ray sources from the INTEGRAL and SWIFT all-sky surveys. The optical data have been obtained with the 1.5-m Russian-Turkish Telescope (RTT-150). Nine X-ray sources have been identified with active galactic nuclei (AGNs). Two of them are located in the nearby spiral galaxies MCG-01-05-047 and NGC 973 seen almost edge-on. One source, IGR J16562-3301, is probably a BL Lac object (blazar). The remaining AGNs are observed as the starlike nuclei of spiral galaxies whose spectra exhibit broad emission lines. The relation between the hard X-ray (17-60 keV) luminosity and the [O III] 5007 line luminosity, $\log L_{\mathrm{X}} / L_{\text {[O III] }} \approx 2.1$, holds good for most of the AGNs detected in hard X rays. However, the luminosities of some AGNs deviate from this relation. The fraction of such objects can reach $\sim 20 \%$. In particular, the [O III] line flux is lower for two nearby edge-on spiral galaxies. This can be explained by the effect of absorption in the galactic disks.

PACS numbers: 95.85.Kr; 97.10.Gz; 97.10.Ri; 97.60.Lf; 98.54.Cm

DOI: $10.1134 / \mathrm{S} 1063773708060017$
\end{abstract}

Key words: X-ray sources, gamma-ray sources, active galactic nuclei, optical observations.

\section{INTRODUCTION}

All-sky surveys in hard X-rays give important information about the populations of objects whose observations are complicated by soft X-ray and optical absorption. The recently completed RXTE (XSS, Revnivtsev et al. 2004) and INTEGRAL (Krivonos et al. 2007) surveys, along with the on-going SWIFT survey (Tueller et al. 2006), are the most sensitive to date.

These surveys cover a large number of known nearby active galactic nuclei (AGNs), cataclysmic variables, and $\mathrm{X}$-ray binaries in the Galactic disk. However, a considerable fraction of the sources, up to $20-30 \%$ of their total number, cannot be identified with previously known objects. To fully exploit the potential of these surveys for statistical studies, one should identify all X-ray sources with optical objects and to determine their type.

The work on optical identifications of hard Xray sources from all-sky surveys was started on the

*E-mail: rodion@hea.iki.rssi.ru 1.5-m Russian-Turkish Telescope (RTT-150) (Bikmaev et al. 2006a, 2006b). This paper presents the results on extragalactic sources, AGNs, obtained in the fall of 2006-spring of 2007. Some of the preliminary data were immediately published in astronomical circulars (Burenin et al. 2006a, 2006b; Mescheryakov et al. 2006; Burenin et al. 2007). Here, we discuss the results of these observations in more detail.

\section{OBSERVATIONS}

The INTEGRAL and SWIFT hard X-ray telescopes are peculiar in that the localization accuracy is, in many cases, insufficient to unambiguously associate an X-ray source with a specific optical object. The error boxes of INTEGRAL and SWIFT hard $\mathrm{X}$-ray sources are typically several arcminutes. During optical observations, a considerable number of galaxies and stars can still fall within such an error box even at high Galactic latitudes. At low Galactic latitudes, a field of such a size contains so many stars that an X-ray source usually cannot be directly identified with a particular optical object. 
Observations in a softer X-ray band, where the positions of sources are measured much more accurately, can be used to improve the positions of hard $\mathrm{X}$-ray sources. These primarily include the data from the ROSAT all-sky survey (Voges et al. 1999). When the $\mathrm{X}$-ray sources are strongly absorbed and are unseen in the ROSAT all-sky survey, the data from the Chandra and XMM-Newton telescopes and the $\mathrm{X}$-ray telescope onboard SWIFT in a harder X-ray band can be used.

The data to improve the INTEGRAL positions of some hard X-ray sources were obtained in dedicated Chandra observations (Sazonov et al. 2005, 2008). An extensive program for observations of SWIFT and INTEGRAL hard X-ray sources is also implemented using the SWIFT X-ray telescope (see, e.g., Tueller et al. 2006). We retrieved all of the necessary additional publicly available data from the HEASARC archive. $^{1}$

For the optical observations, we primarily chose the sources whose positions were improved in one way or another. All of the optical data were obtained with the medium- and low-resolution spectrometer TFOSC ${ }^{2}$ of the 1.5-m Russian-Turkish Telescope (RTT-150). In most cases, we used a slit $54 \mu \mathrm{m}$ in width, which corresponds to 0.96 in the sky, and grism no. 15, which provides the highest quantum efficiency and the widest spectral range (3500-9000 $\AA$ ). In this case, the spectral resolution was $\approx 12 \AA$ (FWHM). Other grisms were used when a higher spectral resolution was required. The data were reduced with the standard IRAF software. ${ }^{3}$

\section{RESULTS}

Table 1 lists the sources identified in the fall of 2006-spring of 2007. The table gives the names, coordinates, $R$ magnitude, types, and redshifts of the sources. The coordinates are given for the epoch J2000 relative to the USNO-B1.0 catalog (Monet et al. 2003). ${ }^{4}$ For nearby galaxies, the coordinates of the active nucleus or the emission maximum at the galactic center, where the optical spectrum was taken, are provided. The $R$ magnitudes are given for reference and were taken from the USNO-B1.0 catalog. The following notation is used for the types of objects: Sy1, 2 denotes a type-1 or-2 Seyfert galaxy and BL Lac denotes a BL Lacertae object. The AGN redshifts $z$ were determined only from narrow lines, such as [O III] 4959, 5007, [NI] 5199, [O I] 6300,

\footnotetext{
${ }^{1}$ http://heasarc.nasa.gov/.

${ }^{2}$ http://astroa.physics.metu.edu.tr/tug/tfosc.html.

${ }^{3}$ http://iraf.noao.edu.

${ }^{4}$ for example, http://archive.eso.org/dss/dss.
}

[S II] 6716, 6731, and, in some cases, [O II] 3727, [Ne III] 3869, and narrow parts of Balmer lines. The error in $z$ does not exceed 0.0001 .

Figure 1 shows the finding charts in which the arrows mark the optical objects identified with hard X-ray sources. For IGR J01528-0326 and IGR J02343+3229, the arrow indicates the position and orientation of the spectrometer slit. The charts show direct images of the X-ray fields in the $r^{\prime}$ filter (SDSS) obtained with the RTT-150 telescope. In the case of IGR J16562-3301, where we were not able to obtain the direct image of sufficient quality, the image taken from DSS-II red plate is shown.

Figure 2 shows the spectra of optical objects used to determine their types. All spectra were reduced to physical fluxes using the observations of spectrophotometric standards from the list by Massey et al. (1988). For the nearby galaxies MCG-0105-047 and NGC 973 (IGR J01528-0326 and IGR $\mathrm{J} 02343+3229$ ), only a small fraction of the flux from their central regions falls into the spectrometer slit. This produces an uncertainty in the photometric calibration of the line fluxes, since the actual angular size of the region in which this emission originates is poorly unknown. The photometric calibration of the spectra for these objects was corrected for the spectrometer slit size by assuming the angular radius of the emitting region to be $9^{\prime \prime}$ and $12^{\prime \prime}$, respectively; the angular sizes were chosen so as to obtain an upper limit for the line fluxes. In the remaining cases, a correction for the slit size was applied by assuming the source to be point-like. This is a good approximation, because all objects have starlike nuclei.

Since the photometric conditions are uncertain during these observations, the photometric calibration may contain large systematic errors, particularly in the blue part of the spectrum, $\lambda<5500 \AA$. The spectra in Fig. 2 were not corrected for interstellar extinction. The spectrum of IGR J16562-3301 is shown in detector counts, because its observations were performed at extremely large zenith angles and the overall shape of its spectrum is severely distorted.

\section{Notes on Individual Objects}

IGR J01528-0326. The source is unambiguously identified with a nearby edge-on spiral galaxy, MCG01-05-047. The optical spectrum for the central part of the galaxy exhibits narrow $\mathrm{H} \alpha$, [N II],6583, and [S II],6716 emission lines. The line intensity ratio $\log ([\mathrm{N} I \mathrm{II}], 6583 / \mathrm{H} \alpha) \approx 0$ suggests that this galaxy most likely has an active nucleus is a type-2 Seyfert (Veilleux and Osterbrock 1987).

IGR J02343+3229. The source is identified with a nearby edge-on spiral galaxy, NGC 973. The 
Table 1. Optical identifications of hard X-ray sources

\begin{tabular}{|c|c|c|c|c|c|c|}
\hline \multirow{2}{*}{ Name } & $\alpha$ & $\delta$ & \multirow{2}{*}{$m_{R}$} & \multirow{2}{*}{ Type } & \multirow{2}{*}{$z$} & \multirow{2}{*}{$\begin{array}{l}\text { Other } \\
\text { name }\end{array}$} \\
\hline & \multicolumn{2}{|c|}{$(\mathrm{J} 2000)$} & & & & \\
\hline IGR J01528-0326 & 015248.90 & -032647.9 & 9.3 & Sy2 & $0.017197^{*}$ & MCG-01-05-047 \\
\hline IGR J02343+3229 & 023420.27 & +323018.6 & 9.8 & Sy2 & $0.016195^{*}$ & NGC 973 \\
\hline IGR J03334+3718 & 033318.78 & +3718 11.4 & 13.5 & Syl & 0.05583 & \\
\hline IGR J13038+5348 & 130359.52 & +534730.2 & 12.9 & Syl & 0.03016 & MCG+09-21-096 \\
\hline IGR J16562-3301 & 165616.77 & -330212.8 & 17.5 & BL Lac & ? & \\
\hline SWIFT J1745.4+2906 & 174538.28 & +2908 22.2 & 14.2 & Syl & 0.11040 & \\
\hline SWIFT J1930.5+3414 & 193013.81 & +341049.8 & 15.8 & Syl & 0.06326 & \\
\hline 1RXS J193347.6+325422 & 193347.16 & +325426.2 & 13.4 & Syl & 0.05794 & \\
\hline RX J2135.9+4728 & 213554.02 & +472822.3 & 13.2 & Syl & 0.02523 & SWIFT J2156.2+4728 \\
\hline
\end{tabular}

* The redshifts were taken from NED: http://nedwww.ipac.caltech.edu/

optical spectrum for the central part of the galaxy exhibits narrow [O III],5007, $\mathrm{H} \alpha,[\mathrm{N} \mathrm{II]}, 6583$, and [S II],6716 emission lines. The line intensity ratios can be estimated as $\log ([\mathrm{O} \mathrm{III}], 5007 / \mathrm{H} \beta)>0.7$ and $\log ([\mathrm{N}$ II $], 6583 / \mathrm{H} \alpha) \approx 0.4$. Thus, this galaxy is a type-2 Seyfert (Veilleux and Osterbrock 1987).

IGR J03334+3718. The source was detected in the ROSAT all-sky survey (1RXS J033316.2+371815), which allows its error circle to be improved to $\approx 10^{\prime \prime}$. Therefore, this source can be unambiguously identified with a galaxy that has a starlike nucleus. The optical spectrum of the galactic nucleus contains redshifted emission linesbroad Balmer lines, narrow [O III] 4959,5007 lines, and others. Hence, it can be classified as a type- 1 Seyfert.

IGR J13038+5348. There are publicly available observations with the SWIFT X-ray telescope for this source (Tueller et al. 2006). Using these data, the position of this source can be determined with an accuracy up to a few arcseconds and it can be unambiguously identified with the bright nearby galaxy MCG+09-21-096. This was previously classified as an emission-line galaxy in the Second Burakan Spectral Survey (Markaryan et al. 19831985; Bicay et al. 2000). The spectrum of this galaxy contains broad Balmer and narrow emission lines, suggesting that this object is a type-1 Seyfert.

IGR J16562-3301. An accurate position of the hard X-ray source was determined from the data of the SWIFT X-ray telescope (Tueller et al. 2006). Using the same $\mathrm{X}$-ray data, we determined a more accurate position of this $\mathrm{X}$-ray source. The systematic error in the astrometric solution was reduced significantly by comparing the positions of two other X- ray sources in the field of the SWIFT telescope, which were unambiguously identified with relatively bright stars in the optical range (Burenin et al. 2007). Thus, we obtain the following coordinates $\alpha$ and $\delta$ of the X-ray source: 16:56:16.82 and -33:02:12.5 (J2000, the astrometric solution was obtained relative to the UCAC2 catalog). Here, the positional error should be almost purely statistical and is about $1^{\prime \prime} .5$.

The position of the very bright radio source NVSS J165616-330211 (with a flux of about 0.4 Jy at $1.4 \mathrm{GHz}$ ) agrees with these coordinates. The coordinates of the only optical object whose position agrees with the positions of both $\mathrm{X}$-ray and radio sources are given in Table 1 . The optical spectrum of this object obtained with the RTT-150 telescope exhibits no intense emission lines (Fig. 2). All these data strongly suggest that this source is a BL Lacertae object. An optical spectrum with a much higher signal-to-noise ratio is required to measure its redshift.

SWIFT J1745.4+2906, SWIFT J1930.5+3414, and 1RXSJ 193347.6+325422. These hard X-ray sources were detected by the SWIFT observatory. The publicly available data from the SWIFT X-ray telescope allow these objects to be unambiguously identified with galactic nuclei. The RTT-150 optical spectra show that they are all type 1 Seyferts (Fig. 2).

SWIFT J2156.2+4728. This object was previously classified as a type-1 Seyfert during the work on optical identifications of X-ray sources from the ROSAT all-sky survey (Motch et al. 1997). However, since these data were not included in the NED database, we also identified it independently. A more accurate value of $z$ than that published by Motch et al. (1997) is given in Table 1. Apart from the galactic 
IGR J01528-0326

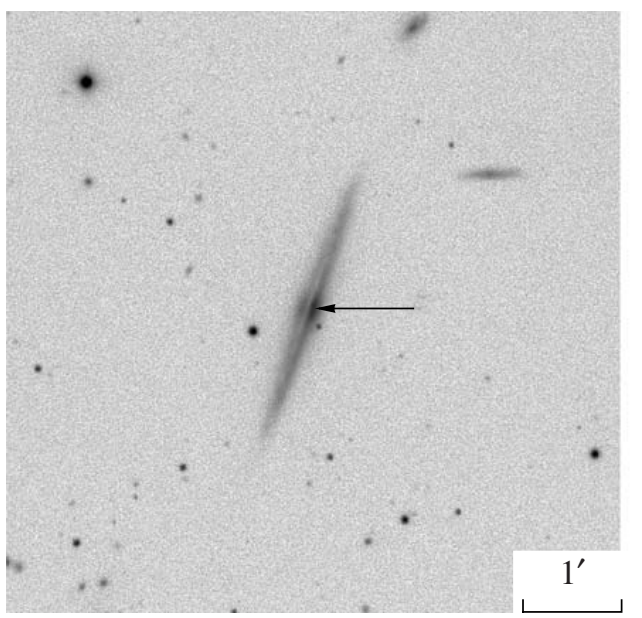

IGR J03334+3718

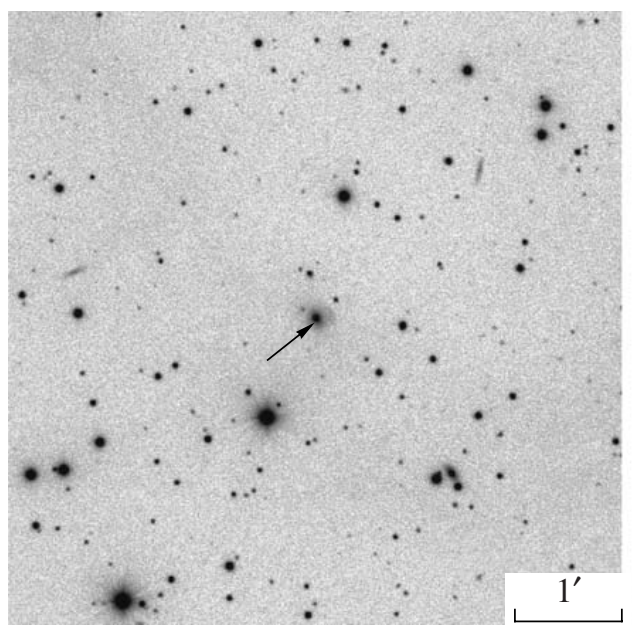

IGR J16562-3301, DSS-II-r

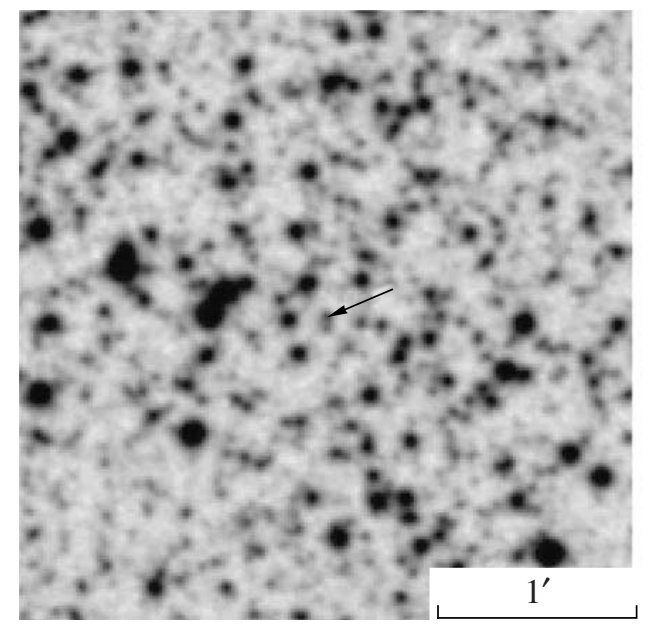

IGR J02343+3229

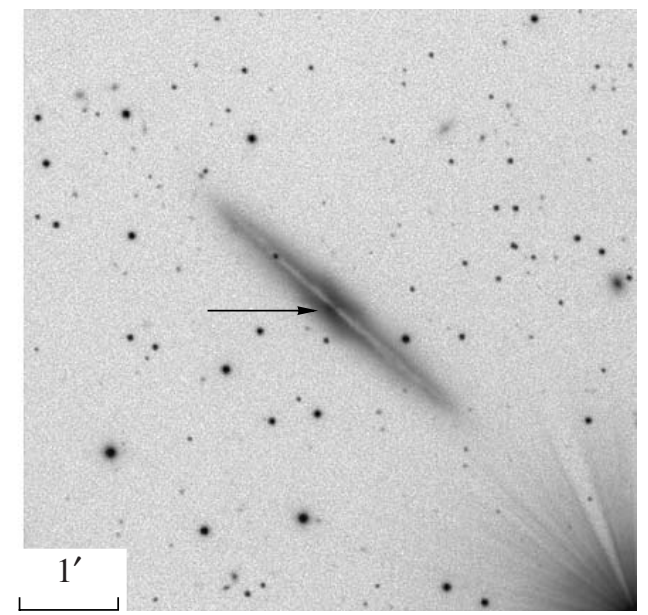

IGR J13038+5348

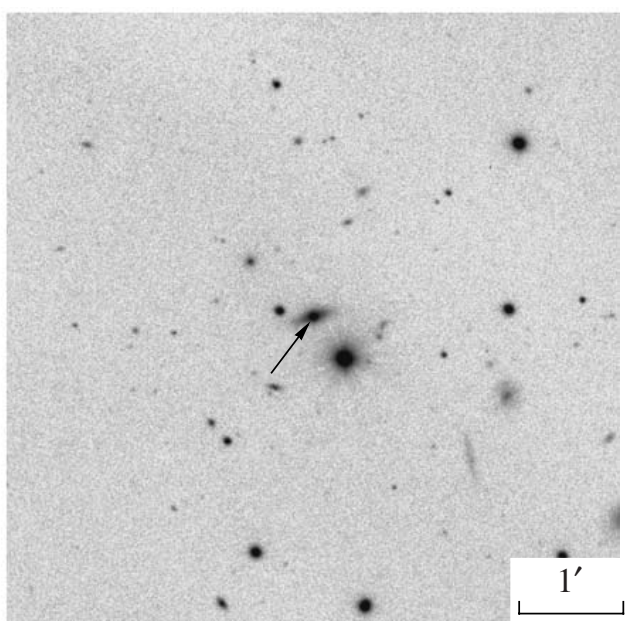

SWIFT J1745.4+2906

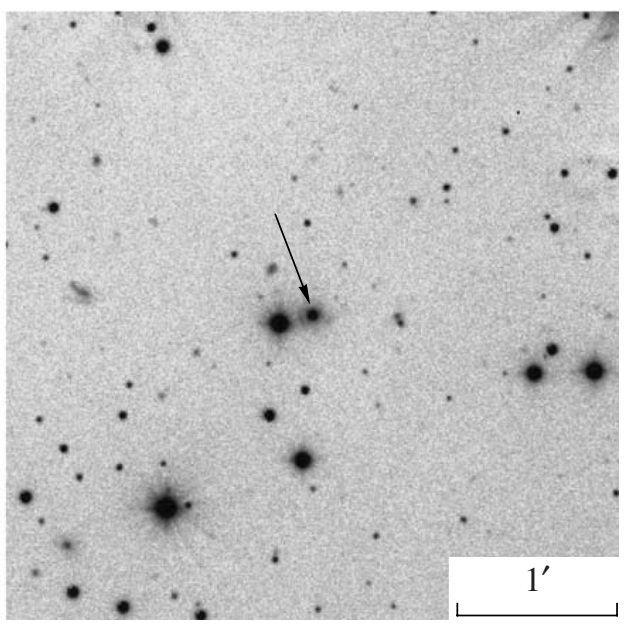

Fig. 1. Optical images of the fields of hard X-ray sources.

nucleus, another starlike object is superimposed on the image of the galaxy (see Fig. 1). We obtained a spectrum of this object and showed that there is a chance projection of a star here. 
SWIFT J1930.5+3414

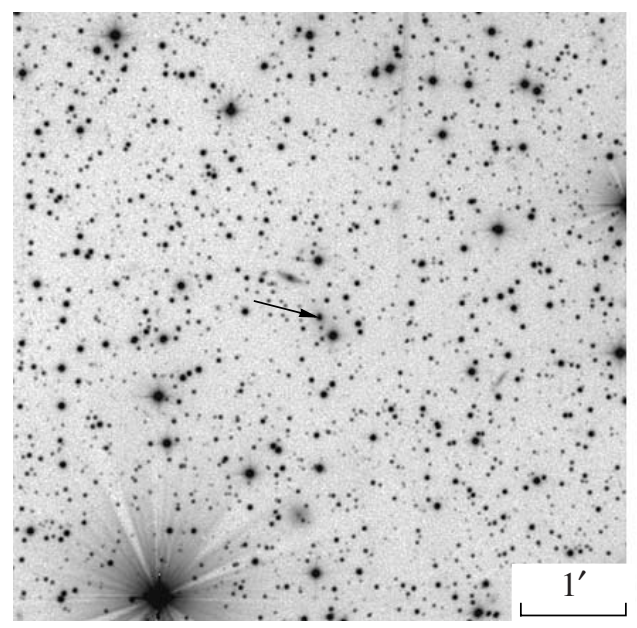

RX J2135.9+4728

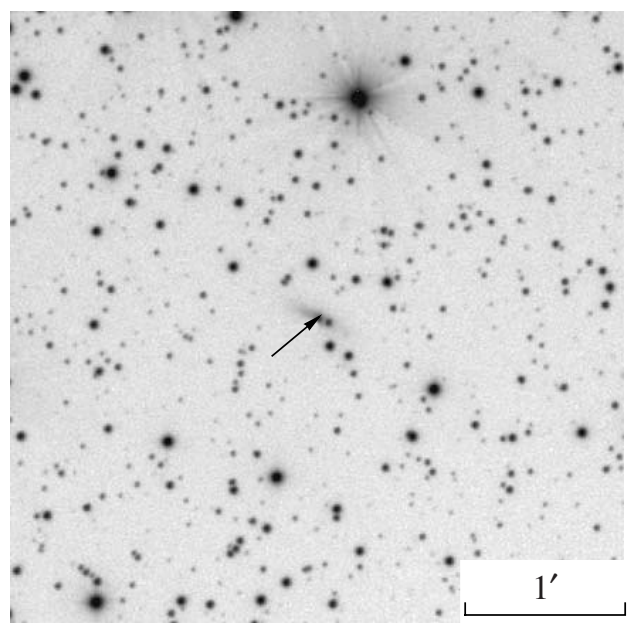

1RXS J193347.6+325422

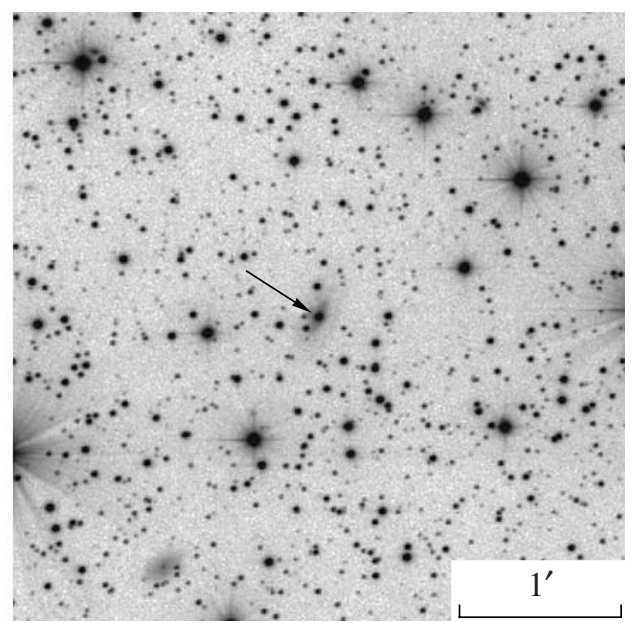

Fig. 1. (Contd.)

\section{DISCUSSION}

The X-ray and [O III],5007 line luminosities ${ }^{5}$ are given in Table 2 . The $\mathrm{X}$-ray fluxes were taken from the catalogs of the INTEGRAL (Krivonos et al. 2007) and SWIFT (Tueller et al. 2006) all-sky surveys. In the latter case, the fluxes were transformed to the 17-60 keV band by assuming a power-law spectrum with photon index $\Gamma=1.8$. Since no data on 1RXS J193347.6+325422 are contained in the current version of the SWIFT catalog, its X-ray flux is not given in Table 2. The [O III],5007 line fluxes were corrected for interstellar extinction using maps from Schlegel et al. (1998).

The relation between the X-ray $17-60 \mathrm{keV}$ and [O III],5007 line luminosities is shown in Fig. 3. The

\footnotetext{
${ }^{5}$ A cosmological model with parameters $\Omega=0.3, \Lambda=0.7$, and $H_{0}=71 \mathrm{~km} \mathrm{~s}^{-1} \mathrm{Mpc}^{-1}$ is used to convert the fluxes into luminosities.
}

data points for AGNs that we detected previously (Bikmaev et al. 2006a) were also added here. Most of the data points fall nicely on the well-known correlation (see, e.g., Heckman et al. 2005). Excluding the three lower points in Fig. 3, we find that the mean value and scatter of the logarithm of the luminosity ratio are $2.13 \pm 0.08$ and 0.27 , respectively, in good agreement with the results for AGNs selected mainly in the optical range (Heckman et al. 2005).

In contrast to the optically selected AGNs, our sample contains AGNs whose [O III] line luminosities are much lower than their X-ray luminosities. These primarily include the two active nuclei in two nearby galaxies seen almost edge-on, MCG01-05-047 and NGC 973 (IGR J01528-0326 and IGR J02343+3229, respectively). As was noted above, there is a significant uncertainty in the calibrations of the spectra for these AGNs related to the uncertainty in the angular size of the emitting 

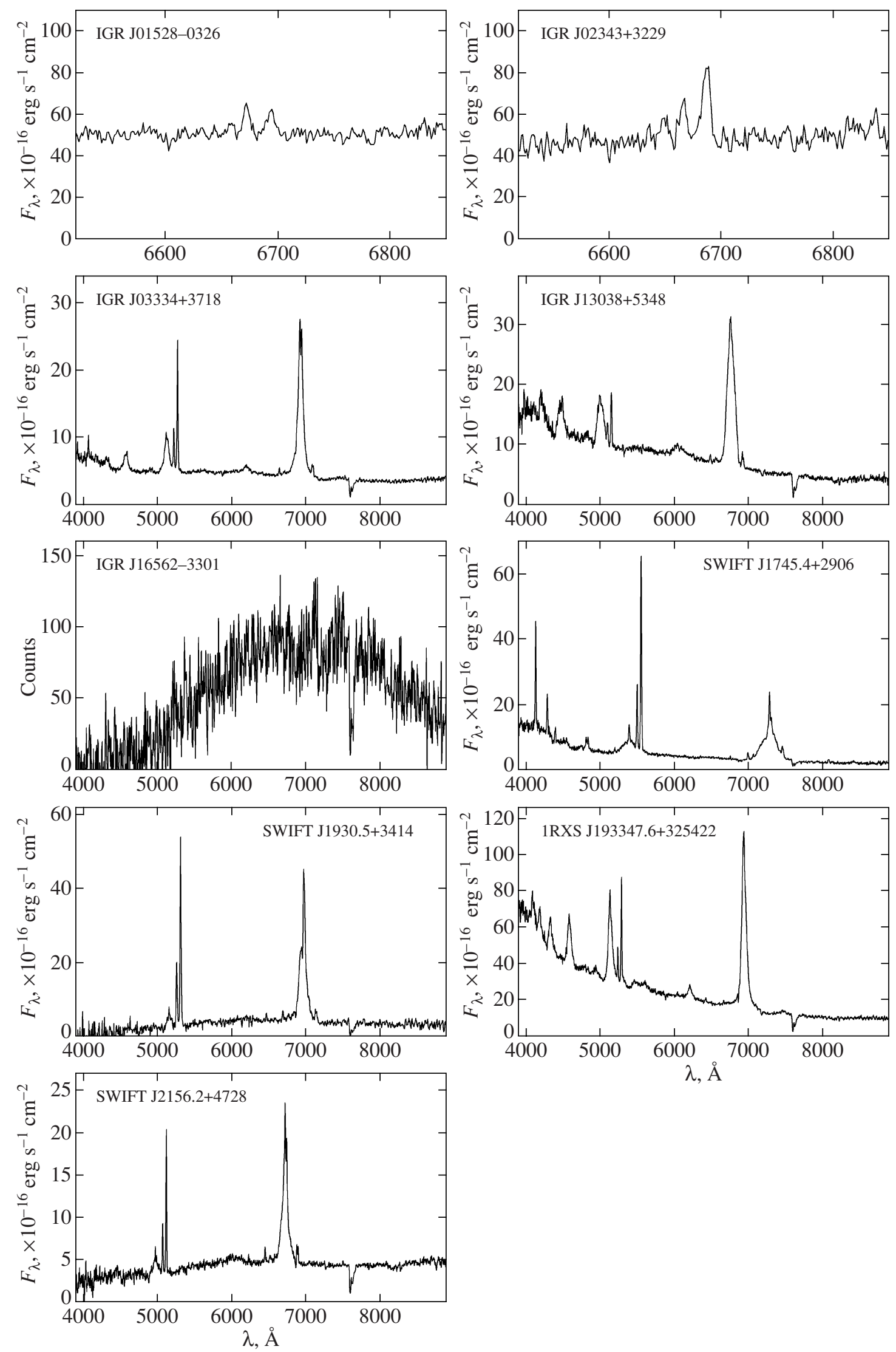

Fig. 2. Spectra of the optical objects identified with hard X-ray sources. 
Table 2. AGN luminosities in the $17-60 \mathrm{keV} \mathrm{X}$-ray band and in the [O III],5007 line

\begin{tabular}{l|c|c}
\hline \multicolumn{1}{c|}{ Name } & $\log L_{\mathrm{X}}$ & $\log L_{[\mathrm{O} \text { III] }}$ \\
\hline IGR J01528-0326 & 43.02 & $<39.08$ \\
IGR J02343+3229 & 43.35 & 40.16 \\
IGR J03334+3718 & 44.15 & 42.05 \\
IGR J13038+5348 & 43.80 & 40.56 \\
SWIFT J1745.4+2906 & 44.71 & 42.50 \\
SWIFT J1930.5+3414 & 44.12 & 42.21 \\
1RXS J193347.6+325422 & - & 42.13 \\
RX J2135.9+4728 & 43.24 & 41.04 \\
\hline
\end{tabular}

region. However, their spectra were calibrated so as to obtain an upper limit for the [O III] line fluxes (see above) and a luminosity deficit in this line should exist in any case. This luminosity deficit can probably be explained by significant absorption in the galactic disk, since both galaxies are seen almost exactly edge-on. Given the observed disk thickness, the size of the absorbed region will not be larger than $\approx 1 \mathrm{kpc}$.

In Fig. 3, the point corresponding to the active nucleus in IGR J13038+5348 deviates noticeably downward. The optical image of this source (Fig. 1)

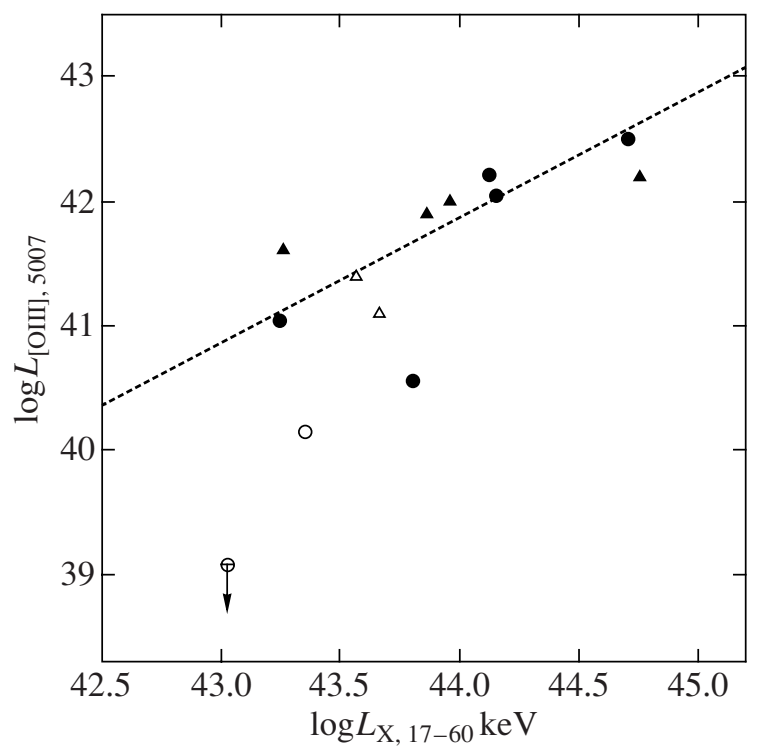

Fig. 3. Relation between the luminosities in the 17$60 \mathrm{keV} \mathrm{X}$-ray band and in the [O III],5007 line. The filled and open symbols indicate Seyfert 1 and 2 galaxies, respectively. The circles and triangles indicate AGNs from this work and those detected previously (Bikmaev et al. 2006a). The dotted line corresponds to $\log L_{\mathrm{X}} / L_{[\mathrm{O} I I]}=2.13$ (see the text). shows that the galaxy here is also seen almost edgeon. However, the spectrum of this object exhibits broad Balmer lines. Some other mechanisms that weaken the narrow forbidden lines may be in action here.

Heckman et al. (2005) conclude that the AGN samples selected by the [O III] flux are as complete as those selected by the X-ray flux. During our work, we encountered several cases where the logarithm of the ratio of the X-ray and [O III] line luminosities differed significantly from $\approx 2.1$. Such objects should distort the AGN selection by the [O III] line flux and the the AGN luminosity function in the [O III] line compared to that in hard $\mathrm{X}$ rays. The fraction of such AGNs can actually be less than $\sim 20 \%$ (as follows from Fig. 3), since we probably consider here only the most strongly absorbed AGNs that were not known previously and were detected on the basis of hard X-ray observations.

\section{CONCLUSIONS}

We presented new results of our optical observations of the fields of unidentified hard X-ray sources detected in statistically complete all-sky surveys. The observations were performed with the $1.5-\mathrm{m}$ Russian-Turkish Telescope (RTT-150). Nine X-ray sources were identified with AGNs. Two of them are located in the nearby spiral galaxies MCG-01-05047 and NGC 973 seen almost edge-on. One source, IGR J16562-3301, is most likely a BL Lac object (blazar). The remaining AGNs are observed as the starlike nuclei of spiral galaxies (Fig. 1) whose spectra exhibit broad emission lines. All of them, except, probably, IGR J16562-3301, are nearby objects-as most of the AGNs in the INTEGRAL survey, they are located at redshifts $z<0.1$. Almost all of the objects lie at high Galactic latitudes, $|b|>5^{\circ}$.

Based on our data obtained previously (Bikmaev et al. 2006a), we showed that the well-known relation $\log L_{\mathrm{X}} / L_{[\mathrm{O} I I I]} \approx 2.1$ between the hard X-ray (17-60 keV) and [O III],5007 line luminosities holds good for most of the AGNs detected in hard X rays. However, a few AGNs deviate greatly from this relation. For example, the [O III] line flux turns out to be lower for the active nuclei in two nearby edgeon spiral galaxies, MCG-01-05-047 and NGC 973. This can be explained by the effect of absorption in the galactic disk. The fraction of AGNs for which the [O III] line fluxes deviate significantly from this relation can reach about $20 \%$. This should correspondingly distort the AGN selection by the [O III] line luminosity and the shape of the AGN luminosity function in the [O III] line compared to that in hard $\mathrm{X}$-rays. 
As a result of recent works on optical identifications of hard X-ray sources (see, e.g., Bikmaev et al. 2006a, 2006b; Masetti et al. 2006, and this paper), the catalog of the INTEGRAL all-sky survey (Krivonos et al. 2007) has become almost complete at high Galactic latitudes. At present, only a few X-ray sources at Galactic latitudes $|b|>5^{\circ}$ remain optically unidentified in this catalog. At the same time, only in the sample of AGNs from this survey, 33 of the 127 objects were not known previously (Sazonov et al. 2007).

Several tens of hard X-ray sources from the INTEGRAL survey at low Galactic latitudes remain optically unidentified. We will continue our work on their optical identification. The optical objects associated with many of the INTEGRAL sources turned out to be too faint for RTT-150. We have submitted an application for the observations of these objects with larger telescopes.

Note. When this paper had already been accepted for publication, the paper by Masetti et al. (2008) appeared in which one of our hard X-ray sources, IGR 16562-3301, was also identified as a BL Lac object (blazar) and its redshift was measured, $z=$ 2.40 .

\section{ACKNOWLEDGMENTS}

This work was supported by the Russian Foundation for Basic Research (project nos. 05-02-16540 and 07-02-01004), the Council of the President of Russia for State Support of Leading Scientific Schools (grants NSh-784.2006.2 and NSh1100.2006.2), and Programs P-04 and OFN-17 of the Russian Academy of Sciences.

\section{REFERENCES}

1. M. D. Bicay, J. A. Stepanian, V. H. Chavushyan, et al., Astron. Astrophys., Suppl. Ser. 147, 169 (2000).

2. I. F. Bikmaev, R. A. Syunyaev, M. G. Revnivtsev, and R. A. Burenin, Pis'ma Astron. Zh. 32, 250 (2006a) [Astron. Lett. 32, 221 (2006a)]; astro-ph/0511405.

3. I. F. Bikmaev, M. G. Revnivtsev, R. A. Burenin, and R. A. Syunyaev, Pis'ma Astron. Zh. 32, 665 (2006b) [Astron. Lett. 32, 588 (2006b)]; astro-ph/0603715.
4. R. Burenin, A. Mescheryakov, M. Revnivtsev, et al., Astron. Telegram 880 (2006a).

5. R. Burenin, A. Mescheryakov, S. Sazonov, et al., Astron. Telegram 883 (2006b).

6. R. Burenin, M. Revnivtsev, A. Mescheryakov, et al., Astron. Telegram 1270 (2007).

7. T. M. Heckman, A. Ptak, A. Hornschemeier, and G. Kauffmann, Astrophys. J. 634, 161 (2005).

8. R. Krivonos, M. Revnivtsev, A. Lutovinov, et al., Astron. Astrophys. 475, 775 (2007).

9. N. Masetti, L. Morelli, E. Palazzi, et al., Astron. Astrophys. 459, 21 (2006).

10. N. Masetti, E. Mason, R. Landi, et al., Astron. Astrophys. (2008) (in press); arXiv:0801.2976.

11. P. Massey, K. Strobel, J. V. Barnes, and E. Anderson, Astrophys. J. 328, 315 (1988).

12. B. T. Makaryan, V. A. Lipovetskiı̌, and D. A. Stepanian, Astrofizika 19, 639 (1983); 20, 21 (1984); 20, 513 (1984); 23, 439 (1985); 25, 345 (1985) [Astrophys. 19, 129 (1983); 20, 113 (1984); 20, 371 (1984).

13. A. Mescheryakov, R. Burenin, S. Sazonov, et al., Astron. Telegram 948 (2006).

14. C. Motch, P. Guillout, F. Haberl, et al., Astron. Astrophys., Suppl. Ser. 122, 201 (1997).

15. D. G. Monet, S. E. Levine, B. Canzian, et al., Astrophys. J. 125, 984 (2003).

16. M. Revnivtsev, S. Sazonov, K. Jahoda, and M. Gilfanov, Astron. Astrophys. 418, 927 (2004).

17. S. Sazonov, E. Churazov, M. Revnivtsev, et al., Astron. Astrophys. 444, L37 (2005); astro-ph/0508593.

18. S. Sazonov, M. Revnivtsev, R. Krivonos, et al., Astron. Astrophys. 462, 57 (2007).

19. S. Sazonov, M. Revnivtsev, R. Burenin, et al., (2008) (in press).

20. D. J. Schlegel, D. P. Finkbeiner, and M. Davis, Astrophys. J. 500, 525 (1998).

21. J. Tueller, C. Markwardt, M. Ajello, et al., Astron. Telegram 835 (2006).

22. J. Tueller, R.F. Mushotzky, S. Barthelmy, et al., (in press); arXiv:0711.4130.

23. S. Veilleux and D. E. Osterbrock, Astrophys. J., Suppl. Ser. 63, 295 (1987).

24. W. Voges, B. Aschenbach, Th. Boller, et al., Astron. Astrophys. 349, 389 (1999).

Translated by A. Serber 